\title{
Tangence
}

\section{L'historien des idées et la littérature personnelle}

\section{Yvan Lamonde}

Numéro 45, octobre 1994

Authenticité et littérature personnelle

URI : https://id.erudit.org/iderudit/025821ar

DOI : https://doi.org/10.7202/025821ar

Aller au sommaire du numéro

Éditeur(s)

Tangence

ISSN

0226-9554 (imprimé)

1710-0305 (numérique)

Découvrir la revue

Citer cet article

Lamonde, Y. (1994). L'historien des idées et la littérature personnelle. Tangence, (45), 10-15. https://doi.org/10.7202/025821ar d'utilisation que vous pouvez consulter en ligne.

https://apropos.erudit.org/fr/usagers/politique-dutilisation/ 


\section{L'historien des idées et la littérature personnelle}

\section{Yvan Lamonde}

La place prise, depuis dix ans, par l'étude de la littérature personnelle ou "intime" ne peut qu'étonner l'historien des idées et lui faire chercher les circonstances et les causes de ce qui est devenu une sorte d'engouement. Cet intérêt pour l'autobiographie, les mémoires, le journal ou les souvenirs a eu l'avantage de se manifester au moment où ces genres attiraient aussi l'attention dans divers pays, en particulier en France où commencaient à paraître les travaux de Philippe Lejeune, qui continue aujourd'hui à inclure les travaux québécois dans ses bilans bibliographiques ${ }^{1}$. Cette littérature a aussi intéressé en même temps historiens et littéraires, comme en témoigne la parution simultanée de l'ouvrage de Françoise van Roey Roux et le mien ${ }^{2}$; du coup, ce corpus de textes gagnait une visibilité scientifique plutôt exceptionnelle. L'attrait pour ces textes au statut problématique s'inscrivait encore dans un contexte de sensibilité à la paralittérature et aux genres non canoniques (science-fiction, téléromans...), à un moment où l'on pouvait avoir l'impression d'un certain piétinement des études littéraires québécoises, engagées dans le systématique des dictionnaires et des répertoires qui pouvait donner à penser à un certain épuisement dans l'analyse des genres classiques.

À un autre niveau, l'histoire socioculturelle du Québec cherchait une trame intellectuelle ou idéologique en scrutant tout autant les institutions (Claude Galarneau), les courants d'idées (Fernand Ouellet, Philippe Sylvain, Pierre Savard) que les formes de la conscience historique (Fernand Dumont). C'est dans ce contexte que je me suis mis à la recherche de textes sériels - conti-

1 Cabiers de sémiotique textuelle, Université de Paris X, nos II (1984-1985), III (1986-1987), IV (1988-1989); Cahiers RITM, nos V (1990-1991); VI (19921993).

2 Françoise van Roey Roux, La littérature intime au Québec, Montréal, Boréal Express, 1983, 256 p.; Yvan Lamonde, Je me souviens. La littérature personnelle au Québec (1880-1980), Québec, Institut québécois de recherche sur la culture, $1983,275 \mathrm{p}$. 
nus dans une longue durée - qui parleraient de la conscience qu'auraient eue des Canadiens français de leur époque, de grands événements ou d'un itinéraire personnel digne de récit. L'heure était à la conscience de soi, tout autant dans un certain milieu québécois des sciences humaines et sociales que dans la société de la Révolution tranquille où le "nous" prenait aussi de plus en plus conscience de lui-même. Paradoxalement toutefois, on était, en Europe, en pleine remise en question du Sujet et en passe, chez Foucault, par exemple, de vider l'homme des Sciences de l'homme. À telle enseigne qu'on pourrait formuler l'hypothèse d'un faible enracinement du structuralisme dans l'appareil méthodologique des praticiens des sciences humaines au Québec.

En un sens, peut-être le phénomène de la parole, de l'expression de soi, de l'énonciation, du dégel langagier associé à la Révolution tranquille a-t-il reflué sur les stratégies de recherche, sur les questionnements nouveaux du passé. Ce qui était alors interrogé l'était à la manière de l'interrogateur interrogé par son propre présent. On se serait alors mis à la recherche d'une préhistoire de cette prise de conscience et de cette prise de parole.

La conscience nouvelle de soi doublée d'un besoin de rattrapage sur le plan de l'expression de soi donnait ainsi peu de prise à l'évacuation du Sujet et facilitait un "retour" du Sujet, qui avait été, tout compte fait, fort peu absent. Alors le néo-libéralisme à allure de post-modernité revenait en force avec le Sujet, avec l'individu et l'individualisme, avec le privé et la privatisation, avec la profusion de "parle, parle, jase, jase", de journaux "intimes" écrits pour les ondes de CBF-FM, d'entertaining intime. Assisterait-on à une banalisation éditoriale des genres de la littérature personnelle en même temps qu'à une certaine inflation de son analyse?

\section{Littérature documentaire et personnelle}

Le réflexe naturel de l'historien est de chercher des détails, des faits et des données dans ces mémoires ou dans ces journaux personnels, un peu comme on a tendance à le faire en regardant les tableaux des Breughel. Cette démarche a sa valeur propre pour autant que celle-ci est perçue comme relative. Mais comme ce sont les acteurs qui écrivent, les témoignages paraissent automatiquement gagner en intérêt et en crédibilité. 
12

Les témoignages valent parfois par leur dimension sérielle. Les souvenirs et journaux relatifs aux rébellions de 1837 et de 1838 ainsi qu'aux expériences d'exil aux États-Unis ou aux Terres australes sont symptomatiquement nombreux et fournissent tout autant des visions et des motivations que des narrations factuelles de ces événements. Un autre corpus peut littéralement révéler un phénomène qu'on n'avait pas identifié autrement. On prendra comme exemple ce groupe de femmes "libérales" du tournant du siècle qui forment une constellation particulièrement intéressante et qu'on retrace dans les journaux d'Henriette Dessaulles, de Lady Lacoste, de Joséphine Marchand-Dandurand ou dans les Quatrevingt ans de souvenirs de Caroline Dessaulles-Béique ${ }^{3}$. Tantôt, ce sont quelques documents qui servent de gué et permettent de traverser une période obscure de l'histoire. On pense, ici, au destin du libéralisme radical après la fermeture de l'Institut canadien de Montréal et la disparition du Pays et à la trame que dessinent Une évocation d'Arthur Buies, les Mémoires du futur sénateur Dandurand et les Mémoires de T.-D. Bouchard. Des événements "fondateurs " comme la grève de l'amiante de 1949 peuvent aussi être lus de points de vue différents, dans les Mémoires du ministre du Travail de l'époque, Antonio Barrette, dans ceux de Gérard Pelletier, correspondant du Devoir sur les lieux de la grève, dans les souvenirs de Gérard Dion, fondateur de l'École des relations industrielles de l'Université Laval ou dans ceux d'un leader syndical de l'époque à Asbestos. Ou alors mémoires, journaux et autobiographies permettent à l'historienne de la condition féminine de chercher une trajectoire dans les écrits de Claire Martin, de Thérèse Casgrain, de Manon Lafleur, de Thérèse Renaud et ou Paule Saint-Onge. L'historien de l'éducation trouvera profit, sur le plan documentaire, à utiliser les souvenirs de collèges ou les monumentaux journaux "personnels" laissés surtout par des prêtres: Arthur Beaudoin, Amédée et David Gosselin, Lionel Groulx, Philéas Fillion, Cyrille Légaré, Michel-Édouard Méthot, André Papineau ou Joseph-Edmond Roy.

L'historien des idées se rendra rapidement compte que ces documents souvent impersonnels à propos de générations d'étudiants et de professeurs peuvent parfois prendre des dimensions plus personnelles et, à tout le moins, devenir le creuset d'une

3 Les auteurs et les récits personnels auxquels il est fait allusion ici sont décrits dans mon ouvrage cité plus haut. 
analyse de l'équipement mental, intellectuel et même émotif de ces hommes (surtout) qui deviendront des clercs, des hommes politiques ou des écrivains. Il suffit parfois de peu de chose pour que la valeur documentaire de ces journaux et souvenirs se transmue en valeur personnelle.

À vingt-cinq ans de distance, je peux évaluer que les questions de départ à l'origine de mes recherches sur la littérature personnelle s'avèrent d'une exceptionnelle richesse analytique et que ces pierres, ramassées çà et là durant deux décennies, constituent, rétrospectivement, un inestimable gué de l'histoire intellectuelle du Québec. J'ai souligné, en introduction à mon. volume, la centralité de Lionel Groulx dans cette constellation de la littérature personnelle québécoise $\mathrm{du} \mathrm{xx}^{\mathrm{e}}$ siècle. La publication ultérieure de son journal et de sa correspondance confirme son importance non seulement dans la pratique des genres de la littérature personnelle mais surtout dans le travail même de la réflexion sur soi d'un homme qui a singulièrement marqué son siècle sur le plan intellectuel. Cette centralité de l'œuvre "personnelle" de Groulx est attestée à la fois par l'importance qu'il a accordée au journal personnel dans la direction spirituelle de ses étudiants - Earl Bartlett, par exemple -, par le recoupement de ses écrits personnels et de ceux d'Armand Lavergne ou de Wilfrid Lebon, à titre d'exemples, et tout autant par les écrits personnels de ceux qui s'opposèrent à lui, en partie ou en totalité. On pense aux Souvenances du père Georges-Henri Lévesque et aux passages qui concernent leur polémique de 1935 autour de l'action catholique et de l'action nationale; au "goupillon" du Refus global; à la double épigraphe de La ligne du risque (1962) de Pierre Vadeboncour, où l'essayiste se sert de Borduas pour faire comprendre que "nous sommes quittes" envers "notre maître, le passé " de Groulx. Sans compter tous ceux qui, d'Asselin à Jean Éthier-Blais ou à Fernand Dumont en passant par André Laurendeau, ont d'une façon ou d'une autre rendu hommage à Groulx.

Mon propos est moins de saluer Groulx que de faire voir comment cette littérature personnelle du $\mathrm{xx}^{\mathrm{e}}$ siècle, dans laquelle il occupe une place prépondérante, met l'historien des idées sur une piste riche et fondamentale. Il y trouve une trajectoire qui part de "notre maître, le passé" et finit dans la ligne du risque, qui court du début du siècle à la Révolution tranquille, qui chemine de l'ACJC à L'action française, à la Crise de civilisation des années trente, à Borduas, à la modernité accomplie. De ce point 
14

de vue, la littérature personnelle est un terreau incomparable pour scruter la genèse des idées, des aspirations, des déceptions; elle est l'humus où poussent les motivations, les désirs, les projets de ceux qui auront été des acteurs de premiers ou de seconds rôles.

Il arrive aussi que l'historien se hérisse à la lecture de mémoires ou de journaux "trop" personnels. La subjectivité l'embête, il ne sait trop que faire de ce témoignage non seulement individuel mais personnel. L'histoire comme science humaine (ou sociale) est ici confrontée à ses limites: comment peut-elle et veut-elle tenir compte de l'humanité de l'homme, du personnel, de la subjectivité ? En quoi le rejet de cette subjectivité est-il plus une solution qu'une démission? L'histoire a un a priori rationaliste, un parti pris positiviste sinon objectiviste. Mais qu'en est-il des "objets" mixtes, des mentalités, des sensibilités?

Les Mémoires de Georges-Émile Lapalme sont, à cet égard, évocateurs parce que riches tout autant par leur style que par leur contenu. C'est lorsqu'on saisit l'amertume dans le ton de ces mémoires que ceux-ci prennent leur réelle signification. Les désillusions ont comme trop faisandé chez ce combattant isolé sous le duplessisme, chez ce reconstructeur du Parti libéral qui voit un nouveau chef plus charismatique venir lui ravir les fruits de la réforme du parti, chez ce guerrier fatigué qui vit les battants des années soixante s'approprier tout le sens de la Révolution tranquille et souffler sur "le vent de l'oubli". Que peut tirer l'historien des idées de ce ton si personnel si ce n'est la réflexion d'un témoin sur ce qu'est le rapport au passé d'un homme et d'une société?

Et puis, l'historien des idées ne fréquente pas impunément cette littérature personnelle. Il court le risque d'être interpellé, d'être touché. Comment, par exemple, lira-t-il Jean-Paul Desbiens alias le Frère Untel, tout autant celui des Insolences [I] que celui de Sous le soleil de la pitié [SSP]? À qui et à quoi pensera l'histotien des idées, et donc l'historien de l'expression, lorsqu'il saisira le sens de ces mots-clés (pour moi!) des Insolences: "Le Canada français : drame de l'expression. (éd. 1960, p. 55) ou cette évocation de la "paralysie de l'expression" (p. 73)? Et, dans Sous le soleil de la pitié (1965): "Dès lors, je me sens tenu d'exprimer ceux dont je suis [ses parents]. Je leur appartiens; je suis la part d'eux-mêmes qui est parvenue à l'expression" (p. 27). Que faut-il 
comprendre de la place de l'expression dans cet itinéraire, de cette détermination à écrire "pour bien établir qu'il est possible de dire ce que l'on pense. ( $I:$ p. 83), de cette insistance pour un Frère, au début des années soixante, à "faire la preuve qu'un chrétien pouvait être baveux" (SSP: p. 64)? Jean-Paul Desbiens a parlé avec les sans-voix, des sans-voix et pour les sans-voix.

Entendement et sensibilité se conjuguent de façon inédite chez Jean-Paul Desbiens; on le perçoit à cette double attention, chez lui, à la pauvreté. À la pauvreté matérielle de l'enfant de la Crise de 1929 qui voit son père pleurer parce que littéralement "éreinté " (SSP: p. 28) et dont la mère attendait qu'il revînt de servir la messe avec son cinq sous pour aller acheter la pinte de lait du déjeuner (SSP: p. 49). Pauvreté intellectuelle aussi, celle de la langue qui est celle de la pensée: on parle joual parce qu'on pense joual ( $I$ : p. 26). L'historien des idées peut aussi trouver chez lui l'ultime dénonciation de l'ultramontanisme: dans les communautés religieuses, "on renonce à l'argent; on renonce à la chair; on ne renonce pas au pouvoir" ( $I$ : p. 68). Puis, au temps où "le goupillon" arrosait tout : "Le profane est sacré: on bénit les ponts et les restaurants, et du même coup, le sacré est profané, puisqu'on le mêle à tout. Assueta vilescunt. En joual, cela veut dire que la familiarité engendre le mépris" ( $I$ : p. 59).

Au contact de la littérature personnelle, l'historien se documente sur le passé plus ou moins intime des autres. Il peut aussi se beurter au passé lorsqu'il sort des sentiers battus des départements d'Histoire, des statistiques, des mandements d'évêques ou des programmes électoraux pour trébucher sur la question de ses propres origines intellectuelles et affectives. Alors l'arbre généalogique ne suffit plus. La "parenté " est autre: avec les idées, avec les formes de la sensibilité, avec les sentiments. L'historien des idées des autres n'a-t-il pas, plus que tout autre, l'obligation scientifique de connaitre l'histoire de ses propres idées? N'a-t-il pas à identifier ce qu'il peut, lui, projeter de rationnel et d'irrationnel sur les autres? Tourné vers la littérature personnelle comme chercheur, n'est-il pas confronté à sa littérature personnelle, perceptible non seulement comme obstacle épistémologique mais aussi comme moyen d'accomplissement scientifique? 